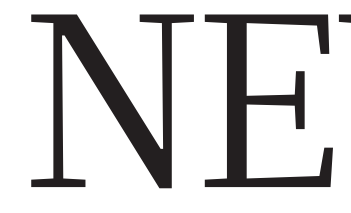

PHYSICS Accelerator closing dooms US bid for Higgs particle p.141

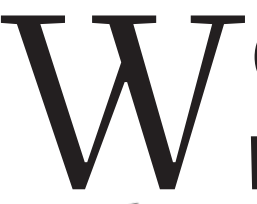
8

ASTRONOMY Planet-hunting telescope finds its first rocky world $\mathbf{p . 1 4 3}$
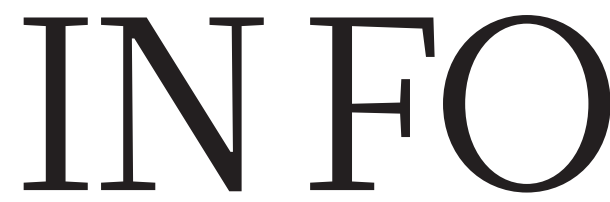

NEUROSCIENCE Can brain imaging diagnose mental health? p.148
ECOLOGY Yellowstone park braces itself for a warmer future p.150

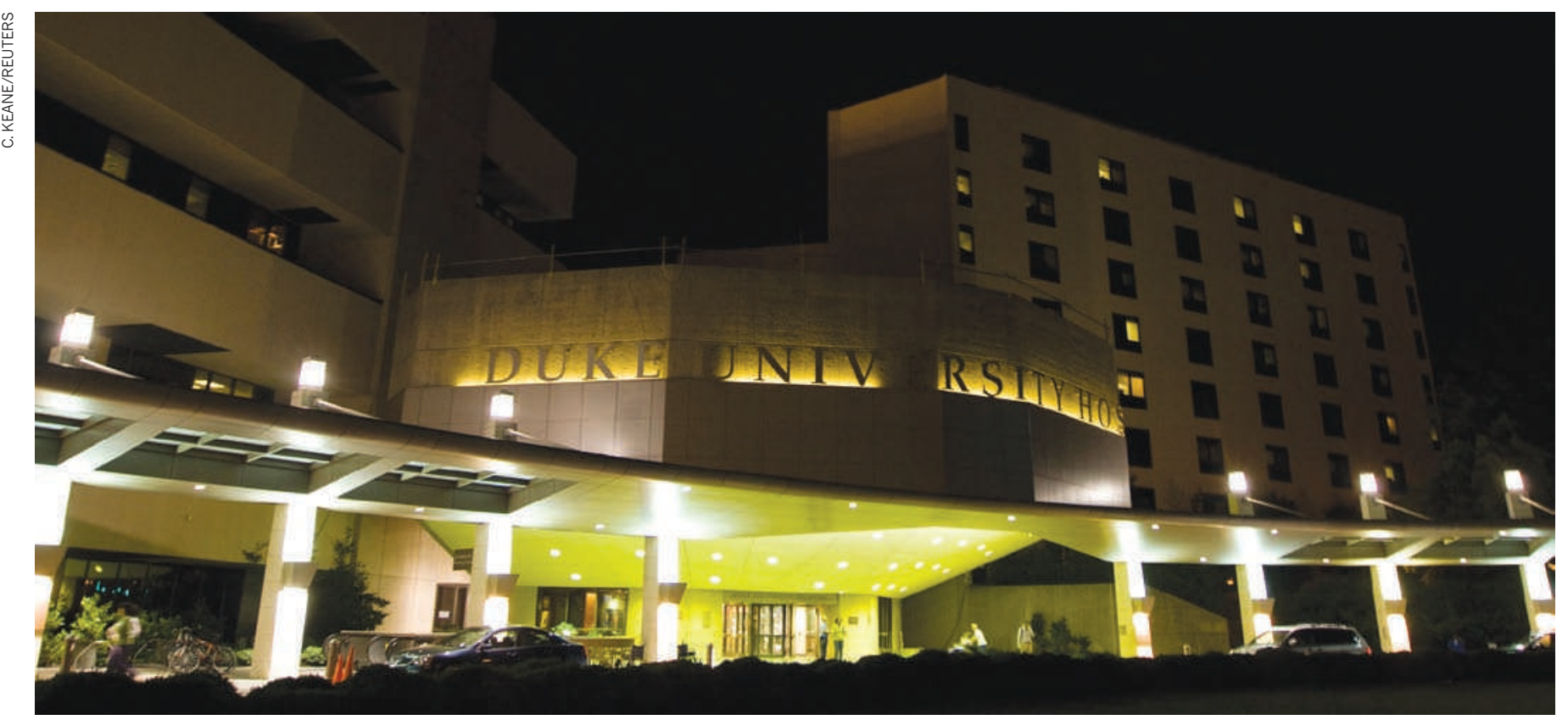

Duke University allowed a controversial set of clinical trials to continue despite serious concerns about the validity of the data on which they were based.

\title{
ETHICS
}

\section{Cancer trial errors revealed}

\section{University officials admit data withheld from review panel before misconduct charges arose.}

\section{BY EUGENIE SAMUEL REICH}

I t was a weekend that Michael Cuffe, vice-president for medical affairs at Duke University in Durham, North Carolina, says he will never forget. It began on 16 July 2010, when Cuffe learned of a damning revelation in The Cancer Letter, a Washington DC-based publication with a reputation for probing controversial topics in cancer research. A story in that day's issue alleged that Anil Potti, a cancer geneticist whose data had been used to design three clinical trials then under way at Duke, had lied on multiple federal grant applications, falsely claiming, among other things, to have been a Rhodes scholar.

That was bad news, but for Cuffe and for Sally Kornbluth, Duke's vice-president for research, that was not the worst of it. Months earlier, the same trials had been suspended after critics raised questions about the underlying science.
Yet Cuffe and Kornbluth had decided to restart them when a review panel seemed to validate Potti's method. The allegations that Potti, who worked at Duke's Institute for Genome Sciences and Policy (ISGP), had padded his CV changed everything. "When it comes to light that someone may have been less than honest in one aspect of their professional life, one begins to wonder whether they have been less than honest in another aspect," says Kornbluth. That weekend, she and Cuffe suspended the trials once again, and initiated a misconduct investigation that is still ongoing. Potti, who could not be reached for comment, resigned from the ISGP in November and took full responsibility for irregularities in his data.

Now, in response to information obtained by Nature under the US $\rightarrow$ NATURE.COM

To read the external review panel's report, visit:

go.nature.com/umtymu
Freedom of Information Act, Kornbluth and Cuffe have offered their account of the mistakes that led the trials to be restarted even after they learned of potential flaws in the underlying data. The affair will have an impact beyond Duke, as the Institute of Medicine, part of the US National Academies in Washington DC, begins to examine research on genome-based patient testing. Originally commissioned to investigate Duke’s controversial trials, the institute's US $\$ 687,000$ study is now expected to focus on providing broader recommendations for the design of clinical trials that similarly use genomic data from individual patients to tailor therapy.

\section{ACADEMIC DISAGREEMENT}

Starting in 2006, Potti and his colleagues at Duke had filed patent applications and published papers $^{1,2}$ describing genomic predictors computer algorithms that take gene-expression data from a cancer cell and predict whether 
- the cancer will be sensitive to a particular therapy (see 'How events unfolded').

Duke began three clinical trials based on the technology, ultimately enrolling around 110 patients with lung or breast cancer. But Potti's work was under fire. Keith Baggerly and Kevin Coombes, biostatisticians at the University of Texas M. D. Anderson Cancer Center in Houston, published a technical comment ${ }^{3}$ and a paper $^{4}$ saying that they had been unable to replicate Potti's findings. As a result, officials at the National Cancer Institute (NCI) in Bethesda, Maryland - which had received a proposal from Duke to start a fourth clinical trial based on Potti's work - contacted the university to request a review. The trials were suspended in September 2009 and a panel of anonymous reviewers, external to Duke, began its work.

While the review was under way, Baggerly and Coombes analysed data that Potti (and his co-authors, none of whom have been implicated in any misconduct) had posted online. The biostatisticians wrote to Kornbluth and Cuffe, pointing out that the data did not match the raw data in the public database from which they were supposedly sourced. "They had numbers with labels that the Duke group said applied, but the labels were wrong," says Baggerly. Yet in December 2009, Duke's review panel concluded that Potti's work was valid, and the trials were restarted.

In an NCI report obtained by Nature, Duke's external reviewers say that they can replicate the results using data provided by Potti, but seem unaware of any doubts about the data. Kornbluth and Cuffe admit that, in consultation with John Harrelson, who was acting as chairman of Duke's Institutional Review Board, they decided not to forward the latest communication from Baggerly and Coombes to the rest of the board or the external reviewers.

Kornbluth denies trying to protect Potti. "Our motivation was not to protect [him], it was to give [him] complete fairness," she says.
They judged that the panel could be improperly biased if it received Baggerly and Coombes's letter, says Cuffe. He and Kornbluth emphasize that, in the early stages of the case, before misconduct allegations were made, they believed that Potti was providing them with "full and truthful answers". But Cuffe acknowledges that the possibility of misconduct may have crossed their minds. "It's not like we had never considered this," he says. If the situation arose again, he says that he would forward "every shred" of evidence to a review panel.

Baggerly regrets that the document wasn't forwarded. "A lot of the back and forth of the past year could have been avoided," he says.

\section{INCREASED SCRUTINY}

Even before Potti's CV padding came to light, doubts about his work were re-emerging. Last April, officials at the NCI determined that they were part-funding the clinical trials through a grant to Potti. NCI documents show that officials weren't reassured by the review panel's report, having found themselves unable to replicate Potti's results using data from the public database. The institute asked Duke to provide it with the information needed for replication, but that process was overtaken by the allegations from The Cancer Letter.

Kornbluth and Cuffe say that Duke is doing its best to learn from the case, and has set up a committee to decide what checks should be in place on basic research before starting clinical trials. Meanwhile, the Institute of Medicine has begun its study, which is expected to last 15 months, and will cover a variety of 'omics-based' tests, including genomics, proteomics and metabolomics.

David Brooks, chief medical officer of Generation Health, a pharmacogenetics testing company in Upper Saddle River, New Jersey, estimates that more than half of cancer clinical trials involve some kind of genomic testing, so the institute's review may have widespread relevance. Richard Simon, a biostatistician at the
$\mathrm{NCI}$, says that one consideration in planning trials is whether genomics data should be used to allocate patients to one therapy or another. An alternative study design is to allocate therapy randomly and have the genomics studied at the same time to see how the two correlate. "It avoids the ethical issues," says Simon.

Back at Duke, other researchers are under scrutiny. Steven McKinney, a statistician at the British Columbia Cancer Research Centre in Vancouver, Canada, has written to the Institute of Medicine to raise the concern, not about misconduct, but that statistical methods used in other Duke studies overlap with a method used by Potti. McKinney told Nature that he is troubled by one study co-authored by Geoffrey Ginsburg, a physician at the ISGP. In the study ${ }^{5}$, approved by Duke's Institutional Review Board and review boards at four further institutions, 57 people were infected with cold or influenza viruses. Gene-expression data were then obtained to see whether it was possible to retrospectively 'predict' which virus they had been infected with - information that could aid the prompt detection of a pandemic. McKinney is concerned that the statistical method being used does not seem to have been proven.

Ginsburg says that "in contrast to the concerns around the cancer work we have gone to great lengths to replicate the results using a variety of statistical methologies". He and his co-authors will look in detail at McKinney's concerns. Kornbluth echoes this sentiment. "This is something we're going to take seriously and look at very closely," she says.

1. Potti, A. et al. Nature Med. 12, 1294-1300 (2006).

2. Hsu, D. S. et al. J. Clin. Oncol. 25, 4350-4357 (2007).

3. Coombes, K. R., Wang, J. \& Baggerly, K. A. Nature Med. 13, 1276-1277 (2007).

4. Baggerly, K. A. \& Coombes, K. R. Ann. Appl. Stat. 3, 1309-1334 (2009).

5. Zaas, A. K. et al. Cell Host Microbe 6, 207-217 (2009).

\section{HOW EVENTS UNFOLDED}

\section{6}

Anil Potti

(pictured), a

cancer geneticist

at Duke University

in Durham,

North Carolina,

and others file

patent applications on the idea of

using gene-expression data to predict

sensitivity to cancer drugs. Potti is

first author on a paper in Nature

Medicine ${ }^{1}$.

\section{7}

Potti is last author on a paper in the Journal of Clinical Oncology (JCO $)^{2}$.

Duke begins three clinical trials to test Potti's predictors in patients with breast or lung cancer.

\section{SEPTEMBER 2009}

Keith Baggerly (pictured) and Kevin Coombes, statisticians at the University of Texas M. D. Anderson Cancer Centre in Houston, publish a paper in Annals of Applied Statistics ${ }^{3}$ stating that they could not replicate Potti's claims. Duke suspends the trials and asks a review panel to investigate.

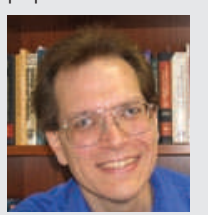

\section{NOVEMBER 2009}

Potti places data underlying the JCO paper online. Baggerly writes to Sally Kornbluth (pictured), Duke vice-dean for research, and Michael Cuffe, Duke vice-president for medical affairs, to point out differences from raw data.
DECEMBER 2009

An unredacted copy of the report by Duke's review panel, later obtained by Nature, shows that the panel replicated Potti's claims using his data, but were unaware that those data contained discrepancies.

\section{JANUARY 2010}

Duke restarts clinical trials.

\section{JUNE 2010}

Harold Varmus (pictured), director of the National Cancer Institute in Bethesda, Maryland, asks the Institute of Medicine to review Duke's trials.
JULY 2010

The Cancer Letter reveals that Potti made false claims about his CV. Trials are suspended and an investigation begins.

\section{NOVEMBER 2010}

JCO paper is retracted. Duke closes the trials permanently. Potti resigns.

\section{DECEMBER 2010}

Institute of Medicine study begins, but will now focus more generally on criteria for genomics predictor.

\section{JANUARY 2011}

Nature Medicine paper is retracted. 


\section{Missing part delays space mission}

Schedule slips for European-led effort to blaze a trail for gravitational-wave detection.

\section{BY EUGENIE SAMUEL REICH}

$\mathrm{F}$ or Stefano Vitale, a principal investigator on the LISA Pathfinder mission, the situation is excruciating. Nearly all the instruments for the $€ 300$-million (US\$400million) spacecraft have been delivered for what was originally to have been a launch this year. But delays have pushed that target to 2013 and possibly later, with everything now held up by a small but crucial component. "All the rest is waiting for one part. It's heartbreaking," says Vitale, a physicist at the University of Trento in Italy.

It is a rougher-than-anticipated start for a mission that was created to find obstacles. LISA Pathfinder is a European-led test of the technology needed to run the Laser Interferometer Space Antenna (LISA), an ambitious effort to detect gravitational waves from sources in the distant Universe. Scientists hope that LISA can achieve this by measuring the precise separations between three pairs of masses free-floating inside three spacecraft positioned 5 million kilometres apart. The technical challenge along with the estimated cost of LISA ( $€ 1$ billion to $€ 2$ billion) made a precursor mission a necessity. If LISA Pathfinder encounters significant problems it could sow doubts about the overall effort.

LISA Pathfinder is not expected to detect gravitational waves, but it must deploy and measure the relative positions of two test masses with sufficient precision for LISA to move forward. The missing piece of the mission is part of a 'caging mechanism' consisting of two sets of eight fingers that will hold the two 1.96-kilogram gold-platinum masses during launch, and then, once the spacecraft reaches its orbit at the L1 Lagrangian point where the gravitational pull of Earth and Sun are balanced, delicately release them. The masses will then float freely inside their separate compartments while the spacecraft uses electrical microthrusters to maintain its position so precisely that the masses do not hit the sides of their containers.

The exacting requirement for a mechanism that can hold the masses firmly enough to withstand a force of 2,000 newtons but still release them without imparting a velocity of more than 5 micrometres per second (18 millimetres an hour) lies at the heart of the delay. A first prototype of the motor powering the fingers failed key tests, prompting the European Space Agency (ESA) to set up a task force to look into the problem. The motor is now being redesigned from scratch. "Little by little, the launch date is slipping," says Pierre Binétruy

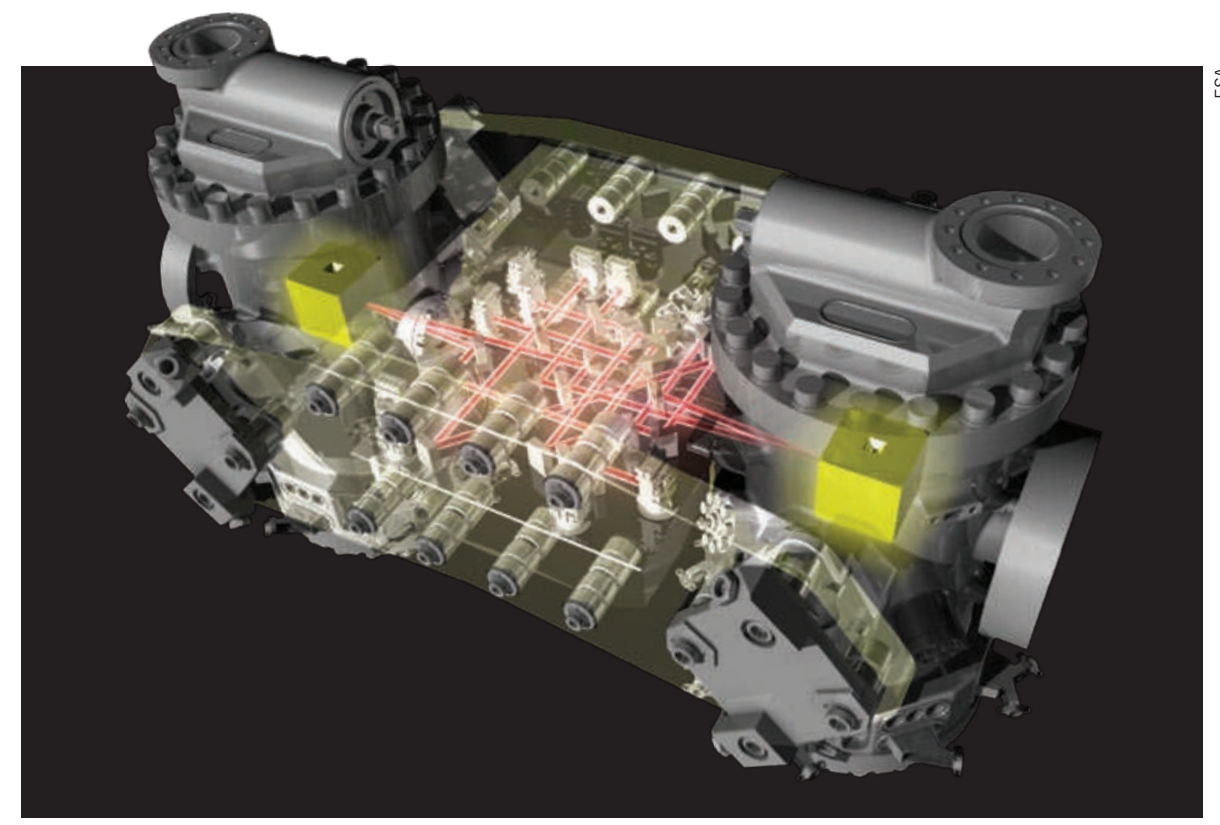

The LISA Pathfinder is missing the mechanism to hold two masses (yellow cubes) in place during launch.

of Paris Diderot University, a physicist on the LISA international science team.

Scientists on the mission say that the important thing is to learn from the delay, to avoid similar problems on LISA. With LISA Pathfinder, ESA initially followed a conventional model for managing space missions, assigning science research groups outside the space agency to design the payload - including the caging mechanism - while industrial partners designed the spacecraft itself. But designers found that the spacecraft was operationally indistinguishable from its science payload, because the positioning of the masses inside it is coupled closely to the craft's ability to keep its place in space using the microthrusters. ESA then took on the design of the caging mechanism together with a contractor, Thales Alenia Space in Milan, Italy, which was unable to comment before Nature went to print.

On 10 February, the ESA Science Programme Committee is expected to assess options for the new design and chart a path forwards.

Last August, the Astro2010 decadal survey of the US National Academy of Sciences ranked participation in LISA among its top priorities, above a competing project, the International $\mathrm{X}$-ray Observatory (IXO). But that recommendation assumed a successful LISA Pathfinder. Xavier Barcons, a physicist at the Cantabria Institute of Physics in Santander, Spain, who works with IXO, says the problems on Pathfinder call into question the decision to rank it higher than his project. "We also have technical difficulties but we've mastered the basics. LISA is a completely new adventure," he says.

He says that it is not clear whether LISA can fly by 2025, as the decadal survey assumed. But Fabio Favata, head of ESA's science coordination office, says that by uncovering problems early, LISA Pathfinder could help LISA avoid delays. "The present situation, although unfortunate, does emphasize the importance of pathfinding," he says. -

\section{CORRECTIONS}

The timeline in the News story 'Cancer trial errors revealed' (Nature 469, 139-140; 2011) stated that Harold Varmus asked the Institute of Medicine to review Duke University's trials in June 2010. He made this request in July 2010.

The News story 'Tevatron faces final curtain' (Nature 469, 141; 2011) states that the Mu2e experiment will study the decay of muons to electrons. In fact, it will look for evidence of neutrino-less conversion of muons to electrons.

The story 'Science fortunes of Balkan neighbours diverge' (Nature 469, 142-143; 2011) wrongly referred to the Romanian Academy of Sciences - it should have said the Romanian Academy. Also Bulgaria cut the budget of its Academy of Sciences by $38 \%$ to 60 million leva (US $\$ 40$ million), not 75 million leva as stated. 